Results The peritoneal case in a 36-year-old man shows a peculiar characteristics long survival (more than 17 years). The patient was treated with cytoreductive surgery and hyperthermic intraperitoneal chemotherapy. Molecular analysis detected multiple chromosomal imbalances. The gains were prevalent. DNA copy number alterations (CNAs) observed loss at 1q21, 8 p 23.1 and gains at 3 p $22.2 \rightarrow 3$ p $22.1, \quad 3$ p $25.3 \rightarrow$ p 25.1 , $4 q 13.1$ and Xq22.2. These are novel CNAs here identified and uncommon in malignant peritoneal mesothelioma.

The pleural case, a 74-year-old man, suffering from a familial Becker type muscular dystrophy, opted for chemotherapy (alimta + cisplatinum) with external hyperthermia. At follow-up in April 2017, about 2 years from diagnosis and 21 months from the beginning of chemotherapy treatments, he has left shoulder pain, general weakness but good respiratory expansion.

Both cases lived in apartments overlooking military barracks from 1963 to 1999.

Discussion Past intensive use of asbestos has implied severe public health consequences among Bari inhabitants. Our study showed that the presence of the asbestos cement factory, as well as the military barracks, have been related with the onset of malignant mesothelioma among the neighbouring resident population.

\section{AVOIDANCE OF UNDER-REPORTING AND SELECTION BIAS IN OCCUPATIONAL INJURY AND ILLNESS SURVEILLANCE SYSTEM}

\footnotetext{
${ }^{1,2}$ Ping Hui Chen*, 1,2Pau Chung Chen. 'Department of Environmental and Occupational Medicine, National Taiwan University Hospital, Taipei, Taiwan; ${ }^{2}$ Institute of Occupational Medicine and Industrial Hygiene, National Taiwan University School of Public Health, Taipei, Taiwan
}

\subsection{6/oemed-2018-ICOHabstracts.405}

Introduction Under-reporting of occupational injury and illness (OII) and accompanied selection bias have always been a difficult challenge for policy making and epidemiological studies in many countries, and each country has come up with various reporting mechanisms to obtain reliable data from different sources. The object of this study is reviewing reporting mechanisms in nine countries, including Taiwan, Japan, Korea, Singapore, Australia, the United States, the United Kingdom, Germany, and France, and concluding a generalised model for constructing an ideal OII surveillance system.

Methods We carry out institutional comparison of various reporting mechanisms in different countries, both quantitatively and qualitatively, based on yearly statistics on OII from each reporting mechanism, and searchable online information, including statutory laws, official webpages, government documents and journal articles, respectively.

Results We categorise various reporting mechanisms into four types, including OSH-Act-based, compensation-based, medicalpractice-based, and health-examination-based, and all countries have more than two types of reporting mechanism. Each type has different reporting incentives, coming from fulfilling statutory reporting obligation by employer, claiming insurance benefit by employee, or seeking information feedback for clinical decision-making and clinical studies by physicians, and thus has its own unique strengths and weaknesses in reporting certain kinds of OII.

Discussion Due to different coverage of OII, each type of reporting mechanism has an irreplaceable role in OII surveillance. Thus, in addition to the operational effectiveness of individual reporting mechanism, emphasis should be put on inclusion of all four types of reporting mechanism with close coordination and integration in constructing an ideal OII surveillance system. To avoid under-reporting and accompanied selection bias, researchers should also get familiar with characteristics and OII coverage of certain reporting mechanism before the utility of reported statistics from that mechanism, and pooling data from more than one reporting mechanisms may be necessary for a more comprehensive picture of OII.

\section{PREVALENCE OF BACK PAIN IN PROFESSIONAL DRIVERS IN ABU DHABI}

Nahid Ebinouff, Omer Alnajeb. Ambulatary Health Services, Abu Dhabi, United Arb Emirates

\subsection{6/oemed-2018-ICOHabstracts.406}

\section{Aims}

1. The principle aim of the study is to examine whether being a driver will lead to an increase in the risk of back pain from the general population by ascertaining the prevalence of back pain among the professional drivers in Abu Dhabi (UAE) and to compare it to the prevalence of back pain in the general population.

2. To investigate whether the type of vehicle driven is a risk factor for back pain by comparing the prevalence of back pain among taxi drivers to the prevalence of back pain among bus drivers and heavy vehicle drivers.

Method It is a cross sectional study that look into the following:

- Prevalence of back pain among professional drivers in Abu Dhabi.

- Comparing the prevalence of back pain between taxi drivers, bus drivers and heavy vehicles.

- All professional drivers attend Al Madina Occupational Health Centre (Abu Dhabi) for all types of medical assessments (Pre-employment, periodic, fitness to return to work or fitness for the extension of services) who agree to participate are included in the study.

- The study ran from January 2015 up to May 2015.

- The sample size was 499.

- Data was collected by a self-filled questionnaire, which has been modified from a validated New Zealand acute low back pain questionnaire (permission taken).

The questionnaire includes general demographic data, work details and back pain information.

Result The study was carried out in Abu Dhabi, UAE. The study population was 499 (the response rate was 92\%). 27 questionnaires were excluded due to incomplete or missed information. The rest were 472 drivers, 141 of them disclose back pain in the previous 3 .

12 month, giving prevalence of $29.9 \%$. From those with back pain $92 \%$ deny any other health problems that affect their back. $82 \%$ of them described their pain as mild. $80.9 \%$ mentioned that minor physical activity does not make their pain worse. $87.2 \%$ declare that, they can do light work for an hour without pain being a problem.

In this study some occupational factors have been investigated with relation to back pain in professional drivers. It shows that, working hours $>8$ hours per day, working days $>5$ days per week and years of driving $>10$ years, are significantly associated with increased back pain in professional 